\title{
Fixed Point Theorems in Non-Archimedean Fuzzy Metric Spaces
}

\author{
Seema Mehra \\ Department of Mathematics, \\ Maharshi Dayanand University, \\ Rohtak-124001 \\ mehra.seema@yahoo.co.in
}

\begin{abstract}
The purpose of this paper is to prove common fixed point theorems in Non- Archimedean fuzzy metric space using the concept of semi-compatibility and weak compatibility of pair of self maps.
\end{abstract}

Keywords: Non- Archimedean fuzzy metric space, common fixed point, semi-compatibility, weak-compatibility.

\section{Introduction}

The concept of fuzzy sets was coined by Zadeh [7] in his seminal paper in 1965. Since then, to use this concept in topology and analysis many authors have expansively developed the theory of fuzzy sets and application. Zadeh [8] anticipated that medical diagnosis would be the most likely application domain of Fuzzy set theory. The theory of fuzzy set is of fundamental importance in Medical diagnosis, effect of drugs, diagnosis process. Recently, a number of fixed point theorems and their applications in fuzzy set theory have been proved by several authors. George and Veeramani[1] and Kramosil and Michalek[6] have introduced the concept of fuzzy metric spaces. The concepts of semi-compatibility and weak-compatibility in fuzzy metric space are given by Singh and Jain [3] which is generalization of commuting and compatible maps. Many authors [2, 4, 11] have proved fixed point theorem in fuzzy(probabilistic) metric spaces. Recently, Dorel Mihet[5] proved a common fixed point theorem in Non- Archimedean fuzzy metric space. The purpose of this paper is to prove common fixed point theorems in Non- Archimedean fuzzy 
metric space using the concept of semi-compatibility and weak compatibility of pair of self maps.

\section{Preliminary Notes}

Definition 2.1 A binary operation $*:[0,1] \times[0,1] \rightarrow[0,1]$

is a continuous $t$-norm if it satisfies the following conditions:

(1) * is associative and commutative,

(2) * is continuous,

(3) a $* 1=$ a for all $\mathrm{a} \in[0,1]$,

(4) $\mathrm{a} * \mathrm{~b} \leq \mathrm{c} * \mathrm{~d}$ whenever $\mathrm{a} \leq \mathrm{c}$ and $\mathrm{b} \leq \mathrm{d}$, for each $\mathrm{a}, \mathrm{b}$,

$\mathrm{c}, \mathrm{d} \in[0,1]$

Two typical examples of continuous t-norm are $\mathrm{a} * \mathrm{~b}=\mathrm{ab}$ and $\mathrm{a} * \mathrm{~b}=\min (\mathrm{a}, \mathrm{b})$.

Definition 2.2. The 3-tuple (X, $\mathrm{M}, *$ ) is called a non-Archimedean fuzzy metric space (shortly, N.A. FM-space) if $X$ is an arbitrary set, * is a continuous $t$-norm and $M$ is a fuzzy set in $X^{2} \times[0, \infty)$ satisfying the following conditions:

For all $\mathrm{x}, \mathrm{y}, \mathrm{z} \in \mathrm{X}$ and $\mathrm{s}, \mathrm{t}>0$,

(NFM-1) $\mathrm{M}(\mathrm{x}, \mathrm{y}, 0)=0$,

(NFM-2) $\mathrm{M}(\mathrm{x}, \mathrm{y}, \mathrm{t})=1$, for all $\mathrm{t}>0$ if and only if $\mathrm{x}=\mathrm{y}$,

(NFM-3) M(x, y, t $)=M(y, x, t)$,

$(\mathrm{NFM}-4) \mathrm{M}(\mathrm{x}, \mathrm{y}, \mathrm{t}) * \mathrm{M}(\mathrm{y}, \mathrm{z}, \mathrm{s}) \leq \mathrm{M}(\mathrm{x}, \mathrm{z}, \max \{\mathrm{t}, \mathrm{s}\})$

Or equivalently $\mathrm{M}(\mathrm{x}, \mathrm{y}, \mathrm{t}) * \mathrm{M}(\mathrm{y}, \mathrm{z}, \mathrm{t}) \leq \mathrm{M}(\mathrm{x}, \mathrm{z}, \mathrm{t})$

(NFM-5) $\mathrm{M}(\mathrm{x}, \mathrm{y},):.[0, \infty) \rightarrow[0,1]$ is left continuous.

Definition 2.3. For $\mathrm{t} \in(0, \infty)$, we define the closed ball $\mathrm{B}[\mathrm{x}, \mathrm{r}, \mathrm{t}]$ with centre $\mathrm{x} \in \mathrm{X}$ and radius $\mathrm{r} \in(0,1)$ as

$\mathrm{B}[\mathrm{x}, \mathrm{r}, \mathrm{t}]=\{\mathrm{y} \in \mathrm{X}, \mathrm{M}(\mathrm{x}, \mathrm{y}, \mathrm{t})>1-\mathrm{r}\}$.

Definition 2.4. Let (X, M, *) be a non-Archimedean fuzzy metric space:

(i) A sequence $\left\{x_{n}\right\}$ in $\mathrm{X}$ is said to be convergent to a point $\mathrm{x} \in \mathrm{X}$ (denoted by

$\left.\lim _{n \rightarrow \infty} x_{n}=x\right)$, if $\lim _{n \rightarrow \infty} M\left(x_{n}, x, t\right)=1$, for all $\mathrm{t}>0$.

(ii) A sequence $\left\{x_{n}\right\}$ in $\mathrm{X}$ is said to be Cauchy sequence if

$\lim$

$n \rightarrow \infty M\left(x_{n+p}, x_{n}, t\right)=1$, for all $\mathrm{t}>0, \mathrm{p}>0$.

(iii) A non-Archimedean fuzzy metric space in which every Cauchy sequence is convergent is said to be complete.

Definition 2.5. A N.A. FM-space (X, M, * ) is said to be of type (C)g if there exists $a \mathrm{~g} \in \Omega$ such that $\mathrm{g}(\mathrm{M}(\mathrm{x}, \mathrm{y}, \mathrm{t})) \leq \mathrm{g}(\mathrm{M}(\mathrm{x}, \mathrm{z}, \mathrm{t}))+\mathrm{g}(\mathrm{M}(\mathrm{z}, \mathrm{y}, \mathrm{t}))$

for all $\mathrm{x}, \mathrm{y}, \mathrm{z} \in \mathrm{X}$ and $\mathrm{t} \geq 0$, where $\Omega=\{\mathrm{g}: \mathrm{g}:[0,1] \rightarrow[0, \infty)$ is continuous, 
strictly decreasing, $\mathrm{g}(1)=0$ and $\mathrm{g}(0)<\infty\}$.

Definition 2.6. A N. A. FM-space (X, M, * ) is said to be of type (D)g if there exists a $\mathrm{g} \in \Omega$ such that $\mathrm{g}(*(\mathrm{~s}, \mathrm{t})) \leq \mathrm{g}(\mathrm{s})+\mathrm{g}(\mathrm{t})$ for all $\mathrm{s}, \mathrm{t} \in[0,1]$.

Definition 2.7. Let $\mathrm{A}$ and $\mathrm{B}$ be mappings from N.A. FM-space (X, M, *) in to itself. The mappings $\mathrm{A}$ and $\mathrm{B}$ are said to be compatible if

$\lim$

$\lim _{n \rightarrow \infty} \mathrm{g}\left(\mathrm{M}\left(\mathrm{ABx}_{\mathrm{n}}, \mathrm{BAx}_{\mathrm{n}}, \mathrm{t}\right)\right)=0$,

for all $\mathrm{t}>0$, whenever $\left\{x_{n}\right\}$ is a sequence in $\mathrm{X}$ such that

$\lim _{n \rightarrow \infty} \mathrm{Ax}_{\mathrm{n}}=\lim _{n \rightarrow \infty} \mathrm{Bx}_{\mathrm{n}}=\mathrm{z}$ for some $\mathrm{z} \in \mathrm{X}$.

Definition 2.8. A pair of maps $\mathrm{A}$ and $\mathrm{B}$ is called weakly compatible pair if they commute at coincidence points i.e., $\mathrm{Ax}=\mathrm{Bx}$ if and only if $\mathrm{ABx}=\mathrm{BAx}$.

Definition 2.9. Let $\mathrm{A}$ and $\mathrm{S}$ be mappings from an N.A. FM-space (X, M,*) into itself. Then the mappings are said to be semi-compatible if

$\lim _{n \rightarrow \infty} \mathrm{g}\left(\mathrm{M}\left(\mathrm{ASx}_{\mathrm{n}}, \mathrm{Sx}, \mathrm{t}\right)\right)=0$, for all $\mathrm{t}>0$, whenever $\left\{x_{n}\right\}$ is a sequence in $\mathrm{X}$

such that

$\lim _{n \rightarrow \infty} \mathrm{Ax}_{\mathrm{n}}=\lim _{n \rightarrow \infty} \mathrm{Bx}_{\mathrm{n}}=\mathrm{x}$ for some $\mathrm{x} \in \mathrm{X}$.

It follows that if $(\mathrm{A}, \mathrm{S})$ is semi-compatible and $\mathrm{Ay}=\mathrm{Sy}$, then $\mathrm{ASy}=\mathrm{SAy}$.

Thus if the pair (A, S) is semi-compatible, then it is weak compatible. The converse is not true as shown in Example 3.2.

\section{Propositions and Lemmas}

These are some propositions and lemmas useful in proving the main results of the paper.

Proposition 3.1 : Let A and S be self-maps on an N.A. FM-space(X, M, * ). If $S$ is continuous, then $(A, S)$ is semi-compatible if and only if $(A, S)$ is compatible.

Proof: Consider a sequence $\left\{x_{n}\right\}$ in $X$ such that $\left\{A x_{n}\right\}$ and $\left\{S_{n}\right\}$ converges to $\mathrm{u} \in \mathrm{X}$. Suppose that $(\mathrm{A}, \mathrm{S})$ is compatible, then $\mathrm{g}\left(\mathrm{M}\left(\mathrm{ASx}_{\mathrm{n}}, \mathrm{Su}, \mathrm{t}\right)\right) \leq \mathrm{g}(\mathrm{M}(\mathrm{ASxn}, \mathrm{SAxn}, \mathrm{t}))+\mathrm{g}(\mathrm{M}(\mathrm{SAxn}, \mathrm{Su} \mathrm{t}))$

letting $\mathrm{n} \rightarrow \infty$, Since $(\mathrm{A}, \mathrm{S})$ is compatible, we have

$\lim$

$\lim _{n \rightarrow \infty} \mathrm{g}\left(\mathrm{M}\left(\mathrm{ASx}_{\mathrm{n}}, \mathrm{Su}, \mathrm{t}\right)\right)=0$. Hence $\mathrm{ASx}_{\mathrm{n}} \rightarrow S u$,i.e., (A,S) is semicompatible.

Conversely, Suppose that $(\mathrm{A}, \mathrm{S})$ is semi-compatible, then

$\mathrm{g}\left(\mathrm{M}\left(\mathrm{ASx}_{\mathrm{n}}, \mathrm{SAx}_{\mathrm{n}}, \mathrm{t}\right)\right) \leq \mathrm{g}\left(\mathrm{M}\left(\mathrm{ASx}_{\mathrm{n}}, \mathrm{Su}, \mathrm{t}\right)\right)+\mathrm{g}\left(\mathrm{M}\left(\mathrm{SAx}_{\mathrm{n}}, \mathrm{Su} \mathrm{t}\right)\right)$.

letting $n \rightarrow \infty$, Since $(A, S)$ is semi-compatible and $S$ is continuous, we have

$\mathrm{SAx}_{\mathrm{n}} \rightarrow \mathrm{Su}$ and this implies 
$\lim _{n \rightarrow \infty} \mathrm{g}(\mathrm{M}(\mathrm{ASxn}, \mathrm{Su}, \mathrm{t}))=0$.

Hence the pair $(\mathrm{A}, \mathrm{S})$ is compatible.

The following is an example of a pair of self-maps $(\mathrm{A}, \mathrm{S})$ which is compatible but not semi-compatible. Further, it is also seen here that the semi-compatibility of the pair (A, S) need not imply the semi-compatibility of (S,A).

Example 3.2: Let $X=[0,1]$ and let $(X, M, *)$ be the N.A. FM-space with $\mathrm{g}(\mathrm{M}(\mathrm{x}, \mathrm{y}, \mathrm{t}))=\mathrm{g}\left[\exp \frac{|x-y|}{t}\right]^{-1}$ for all $\mathrm{x}, \mathrm{y} \in \mathrm{X}, \mathrm{t}>0$. Define self-map $\mathrm{S}$ as follows:

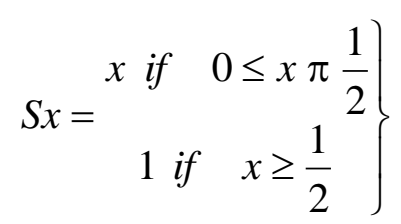

Let $\mathrm{I}$ be the identity map on $\mathrm{X}$ and $x_{n}=\frac{1}{2}-\frac{1}{n}$. Then, $\left\{\mathrm{Ix}_{\mathrm{n}}\right\}=\left\{\mathrm{X}_{\mathrm{n}}\right\} \rightarrow \frac{1}{2}$ And $\left\{\mathrm{Sx}_{\mathrm{n}}\right\}=\left\{\mathrm{x}_{\mathrm{n}}\right\} \rightarrow \frac{1}{2}$. Thus $\left\{\mathrm{ISx}_{\mathrm{n}}\right\}=\left\{\mathrm{Sx}_{\mathrm{n}}\right\} \rightarrow \frac{1}{2} \neq \mathrm{S}\left(\frac{1}{2}\right)$. Hence $(\mathrm{I}, \mathrm{S})$ is not semicompatible.

Again as (I, S) is commuting, it is compatible. Futher, for any sequence $\left\{x_{n}\right\}$ in $X$ such that $\left\{\mathrm{x}_{\mathrm{n}}\right\} \rightarrow \mathrm{x}$, we have $\left\{\mathrm{SIx}_{\mathrm{n}}\right\}=\left\{\mathrm{Sx}_{\mathrm{n}}\right\} \rightarrow \mathrm{x}=\mathrm{Ix}$. Hence (S, I) is always semicompatible.

Remark 3.3: The above example gives an important aspect of semicompatibility as the pair of self-maps (I,S) is commuting, hence it is weakly commuting, compatible and weak compatible yet it is not semi-compatible. Further, it is to be noted that the pair $(\mathrm{S}, \mathrm{I})$ is semi-compatible but $(\mathrm{I}, \mathrm{S})$ is not semi-compatible here. The following is an example of a pair of self-maps (A, S) which is semicompatible but not compatible.

Example 3.4 : Let $(\mathrm{X}, \mathrm{M}, *)$ be the N.A. FM-space, where $\mathrm{X}=[0,2]$, with $\mathrm{t}$ norm defined by $a * b=\min \{a, b\}$, for all $a, b \in[0,1]$ and $\mathrm{g}(\mathrm{M}(\mathrm{x}, \mathrm{y}, \mathrm{t}))=\mathrm{g}\left(\frac{t}{t+d(x, y)}\right)$ for all $\mathrm{t}>0$ and $\mathrm{g}(\mathrm{M}(\mathrm{x}, \mathrm{y}, 0))<\infty$, for all $\mathrm{x}, \mathrm{y} \in \mathrm{X}$.

Define self-maps A and S on X follows : 
$\mathrm{Ax}=\left\{\begin{array}{l}2 \text { if } 0 \leq x \leq 1 \\ \frac{x}{2} \text { if } 1 \pi x \leq \frac{1}{2}\end{array}, \mathrm{Sx}=\left\{\begin{array}{c}2 \text { if } x=1 \\ \frac{x+3}{5} \text { otherwise }\end{array}\right.\right.$

And $x_{n}=2-\frac{1}{2 n}$. Then we have $\mathrm{S}(1)=\mathrm{A}(1)=2$ and $\mathrm{S}(2)=\mathrm{A}(2)=1$. Also

$\mathrm{SA}(1)=\mathrm{AS}(1)=1$ and $\mathrm{SA}(2)=\mathrm{AS}(2)=2$. Thus $(\mathrm{A}, \mathrm{S})$ is weak compatible.

Again,

$\mathrm{Ax}_{\mathrm{n}}=1-\frac{1}{4 n}, \mathrm{Sx}_{\mathrm{n}}=1-\frac{1}{10 n}$.

Thus, $A x_{n} \rightarrow 1, S x_{n} \rightarrow 1$.

Hence $\mathrm{u}=1$.

Further,

$\operatorname{SAx}_{\mathrm{n}}=\frac{4}{5}-\frac{1}{20 n}, \mathrm{ASx}_{\mathrm{n}}=2$

Now,

$\lim _{n \rightarrow \infty} \mathrm{g}\left(\mathrm{M}\left(\mathrm{ASx}_{\mathrm{n}}, \mathrm{Su}, \mathrm{t}\right)\right)=\mathrm{g}(\mathrm{M}(2,2, \mathrm{t}))=0$,

$\lim _{n \rightarrow \infty} \mathrm{g}\left(\mathrm{M}\left(\mathrm{ASx}_{\mathrm{n}}, \mathrm{SAx}_{\mathrm{n}}, \mathrm{t}\right)\right)=\lim _{n \rightarrow \infty} \mathrm{g}\left(\mathrm{M}\left(2, \frac{4}{5}-\frac{1}{20 n}, \mathrm{t}\right)\right)=\mathrm{g}\left(\frac{t}{t+\frac{6}{5}}\right) \neq 0$, for all

$t>0$. Hence $(\mathrm{A}, \mathrm{S})$ is semicompatible but it is not compatible.

Remark 3.5: (1) If a N.A. FM-space $(X, M, *)$ is of type $(D)_{g}$ then $(X, M, *)$ is of type $(\mathrm{C})_{\mathrm{g}}$.

(2) If $(\mathrm{X}, \mathrm{M}, *)$ is a N.A. FM-space and $* \geq *_{\mathrm{m}}$, where $*_{\mathrm{m}}(\mathrm{s}, \mathrm{t})=$ $\max \{\mathrm{s}+\mathrm{t}-1,0\}$, then $(\mathrm{X}, \mathrm{M}, *)$ is of type $(\mathrm{D})_{\mathrm{g}}$ for $\mathrm{g} \in \Omega$ defined by $\mathrm{g}(\mathrm{t})=1-\mathrm{t}$.

Throughout this paper, let (X,M,* ) be a complete N.A.FM-space of type (D)g with a continuous strictly increasing t-norm $*$.

Let $\phi:[0,+\infty) \rightarrow[0,+\infty)$ be a function satisfying the following condition $(\Phi)$ :

$(\Phi) \phi$ is upper semi-continuous from the right and $\phi(\mathrm{t})<\mathrm{t}$ for all $\mathrm{t}>0$. 
Lemma 3.6[10] : If a function $\phi:[0,+\infty) \rightarrow[0,+\infty)$ satisfies the condition $(\Phi)$, then we have

(1) For all $\mathrm{t} \geq 0, \lim _{\mathrm{n} \rightarrow \infty} \phi^{\mathrm{n}}(\mathrm{t})=0$, where $\phi^{\mathrm{n}}(\mathrm{t})$ is the $\mathrm{n}$-th iteration of $\phi(\mathrm{t})$.

(2) If $\left\{t_{n}\right\}$ is a non-decreasing sequence of real numbers and $t_{n+1} \leq \phi\left(t_{n}\right), n=1,2, \ldots$, then $\lim _{n \rightarrow \infty} t_{n}=0$. In particular, if $t \leq \phi(t)$ for all $t \geq 0$, then $t=0$.

Lemma 3.7[9]: Let $\left\{y_{n}\right\}$ be a sequence in $X$ such that $\lim _{n \rightarrow \infty} F\left(y_{n}, y_{n+1}, t\right)=1$ for all $t>0$. If the sequence $\left\{y_{n}\right\}$ is not a Cauchy sequence in $X$, then there exist $\in_{0}>0$, $\mathrm{t}_{0}>0$, two sequences $\left\{\mathrm{m}_{\mathrm{i}}\right\},\left\{\mathrm{n}_{\mathrm{i}}\right\}$ of positive integers such that

(A) $\mathrm{m}_{\mathrm{i}}>\mathrm{n}_{\mathrm{i}}+1$, and $\mathrm{n}_{\mathrm{i}} \rightarrow \infty$ as $\mathrm{i} \rightarrow \infty$,

(B) $\mathrm{F}\left(y_{m_{i}}, y_{n_{i}}, \mathrm{t}_{0}\right)<1-\epsilon_{0}$ and $\mathrm{F}\left(y_{m_{i}-1}, y_{n_{i}}, \mathrm{t}_{0}\right) \geq 1-\epsilon_{0}, \mathrm{i}=1,2, \ldots$

\section{Main Results}

These are the main results of the paper.

Theorem 4.1 Let $\mathrm{A}, \mathrm{B}, \mathrm{S}, \mathrm{T}: \mathrm{X} \rightarrow \mathrm{X}$ be mappings such that

(1) $\mathrm{A}(\mathrm{X}) \subset \mathrm{T}(\mathrm{X})$ and $\mathrm{B}(\mathrm{X}) \subset \mathrm{S}(\mathrm{X})$,

(2) $\mathrm{g}(\mathrm{M}(\mathrm{Ax}, \mathrm{By}, \mathrm{t})) \leq \phi(\max \{\mathrm{g}(\mathrm{M}(\mathrm{Sx}, \mathrm{Ty}, \mathrm{t})), \mathrm{M}(\mathrm{Sx}, \mathrm{Ax}, \mathrm{t})), \mathrm{g}(\mathrm{M}(\mathrm{Ty}, \mathrm{By}, \mathrm{t}))$,

$1 / 2(\mathrm{~g}(\mathrm{M}(\mathrm{Sx}, \mathrm{By}, \mathrm{t}))+\mathrm{g}(\mathrm{M}(\mathrm{Ty}, \mathrm{Ax}, \mathrm{t})))\})$ for all $\mathrm{t}>0$,

where a function $\phi:[0, \infty) \rightarrow[0, \infty)$ satisfies the condition $(\Phi)$.

(3) the pair $(\mathrm{A}, \mathrm{S})$ is semi-compatible and $(\mathrm{B}, \mathrm{T})$ is weakly compatible

(4) one of $\mathrm{A}$ or $\mathrm{S}$ is continuous

Then $\mathrm{A}, \mathrm{B}, \mathrm{S}$ and $\mathrm{T}$ have a uniqe common fixed point in $\mathrm{X}$.

Proof: Let $\mathrm{x}_{0} \in \mathrm{X}$, then by (1), there exists a point $\mathrm{x}_{1} \in \mathrm{X}$ such that $\mathrm{Ax}_{0}=\mathrm{Tx}_{1}$, since $\mathrm{A}(\mathrm{X}) \subset \mathrm{T}(\mathrm{X})$. Since $\mathrm{B}(\mathrm{X}) \subset \mathrm{S}(\mathrm{X})$, for this point $\mathrm{x}_{1}$, we can choose a point $\mathrm{X}_{2}$ $\in \mathrm{X}$ such that $\mathrm{Bx}_{1}=\mathrm{Sx}_{2}$ and so on. Inductively, we can define a sequence $\left\{\mathrm{y}_{\mathrm{n}}\right\}$ in $\mathrm{X}$ such that

(5) $\mathrm{y}_{2 \mathrm{n}}=\mathrm{Ax}_{2 \mathrm{n}}=\mathrm{Tx}_{2 \mathrm{n}+1}$ and $\mathrm{y}_{2 \mathrm{n}+1}=\mathrm{Sx}_{2 \mathrm{n}+2}=\mathrm{Bx}_{2 \mathrm{n}+1}$ for $\mathrm{n}=0,1,2 \ldots$.

Now we prove the sequence $\left\{\mathrm{y}_{\mathrm{n}}\right\}$, defined by (5), such that

$\lim _{\mathrm{n} \rightarrow \infty} \mathrm{g}\left(\mathrm{M}\left(\mathrm{y}_{\mathrm{n}}, \mathrm{y}_{\mathrm{n}+1}, \mathrm{t}\right)\right)=0$ for all $\mathrm{t}>0$ is a Cauchy sequence in $\mathrm{X}$.

Since $g \in \Omega$, it follows that $\lim _{\mathrm{n} \rightarrow \infty} \mathrm{M}\left(\mathrm{y}_{\mathrm{n}}, \mathrm{y}_{\mathrm{n}+1}, \mathrm{t}\right)=1$ for all $t>0$ if and only if $\lim _{\mathrm{n} \rightarrow \infty} \mathrm{g}\left(\mathrm{M}\left(\mathrm{y}_{\mathrm{n}}, \mathrm{y}_{\mathrm{n}+1}, \mathrm{t}\right)\right)=0$ for all $t>0$. By Lemma 3.7, if $\left\{\mathrm{y}_{\mathrm{n}}\right\}$ is not a Cauchy sequence in $X$, then there exist $\in_{0}>0, \mathrm{t}_{0}>0$ and two sequences $\left\{\mathrm{m}_{\mathrm{i}}\right\},\left\{\mathrm{n}_{\mathrm{i}}\right\}$ of positive integers such that

(A) $\mathrm{m}_{\mathrm{i}}>\mathrm{n}_{\mathrm{i}}+1$ and $\mathrm{n}_{\mathrm{i}} \rightarrow \infty$ as $\mathrm{i} \rightarrow \infty$,

(B) $\mathrm{g}\left(\mathrm{M}\left(\mathrm{ym}_{\mathrm{i}}, \mathrm{yn}_{\mathrm{i}}, \mathrm{t}_{0}\right)\right)>\mathrm{g}\left(1-\epsilon_{0}\right)$ and $\mathrm{g}\left(\mathrm{M}\left(\mathrm{ym}_{\mathrm{i}-1}, \mathrm{yn}_{\mathrm{i}}, \mathrm{t}_{0}\right)\right) \leq \mathrm{g}\left(1-\epsilon_{0}\right), \mathrm{i}=1,2, \ldots$.

Thus we have

$\mathrm{g}\left(1-\in_{0}\right)<\mathrm{g}\left(\mathrm{M}\left(y_{m_{i}}, y_{n_{i}}, \mathrm{t}_{0}\right)\right)$

$$
\leq \mathrm{g}\left(\mathrm{M}\left(y_{m_{i}}, y_{m_{i}-1}, \mathrm{t}_{0}\right)\right)+\mathrm{g}\left(\mathrm{M}\left(y_{m_{i}-1}, y_{n_{i}}, \mathrm{t}_{0}\right)\right)
$$




$$
\leq \mathrm{g}\left(\mathrm{M}\left(y_{m_{i}}, y_{m_{i}-1}, \mathrm{t}_{0}\right)\right)+\mathrm{g}\left(1-\varepsilon_{0}\right)
$$

Letting $\mathrm{i} \rightarrow \infty$ in (6), we have

(7) $\lim _{\mathrm{n} \rightarrow \infty} \mathrm{g}\left(\mathrm{M}\left(y_{m_{i}}, y_{n_{i}}, \mathrm{t}_{0}\right)\right)=\mathrm{g}\left(1-\in_{0}\right)$.

On the other hand, we have $\mathrm{g}\left(1-\epsilon_{0}\right)<\mathrm{g}\left(\mathrm{M}\left(\mathrm{ym}_{\mathrm{i}}, \mathrm{yn}_{\mathrm{i}}, \mathrm{t}_{0}\right)\right)$

(8) $\quad \leq \mathrm{g}\left(\mathrm{M}\left(y_{n_{i}}, y_{n_{i}+1}, \mathrm{t}_{0}\right)\right)+\mathrm{g}\left(\mathrm{M}\left(y_{n_{i}+1}, y_{m_{i}}, \mathrm{t}_{0}\right)\right)$

Now, consider $\mathrm{g}\left(\mathrm{M}\left(y_{n_{i}+1}, y_{m_{i}}, \mathrm{t}_{0}\right)\right)$ in (8). Without loss of generality assume that both $n_{i}$ and $m_{i}$ are even.

Then, by (2), we have

$$
\begin{aligned}
& \mathrm{g}\left(\mathrm{M}\left(y_{n_{i}+1}, y_{m_{i}}, \mathrm{t}_{0}\right)\right)= \mathrm{g}\left(\mathrm{M}\left(\mathrm{A} x_{m_{i}}, \mathrm{~B} x_{n_{i}+1}, \mathrm{t}_{0}\right)\right) \\
& \leq \phi\left(\operatorname { m a x } \left\{\mathrm{g}\left(\mathrm{M}\left(\mathrm{S} x_{m_{i}}, \mathrm{~T} x_{n_{i}+1}, \mathrm{t}_{0}\right)\right),\right.\right. \\
& \mathrm{g}\left(\mathrm{M}\left(\mathrm{S} x_{m_{i}}, \mathrm{~A} x_{m_{i}}, \mathrm{t}_{0}\right)\right), \mathrm{g}\left(\mathrm{M}\left(\mathrm{T} x_{n_{i}+1}, \mathrm{~B} x_{n_{i}+1}, \mathrm{t}_{0}\right)\right), \\
&\left.\left.1 / 2\left(\mathrm{~g}\left(\mathrm{M}\left(\mathrm{S} x_{m_{i}}, \mathrm{~B} x_{n_{i}+1}, \mathrm{t}_{0}\right)\right)+\mathrm{g}\left(\mathrm{M}\left(\mathrm{T} x_{n_{i}+1}, \mathrm{~A} x_{m_{i}}, \mathrm{t}_{0}\right)\right)\right)\right\}\right) \\
&(9)=\phi\left(\operatorname { m a x } \left\{\mathrm{g}\left(\mathrm{M}\left(y_{m_{i}-1}, y_{n_{i}}, \mathrm{t}_{0}\right)\right), \mathrm{g}\left(\mathrm{M}\left(y_{m_{i}-1}, y_{m_{i}}, \mathrm{t}_{0}\right)\right), \mathrm{g}\left(\mathrm{M}\left(y_{n_{i}}, y_{n_{i}+1}, \mathrm{t}_{0}\right)\right),\right.\right. \\
&\left.\left.1 / 2\left(\mathrm{~g}\left(\mathrm{M}\left(y_{m_{i}-1}, y_{n_{i}+1}, \mathrm{t}_{0}\right)\right)+\mathrm{g}\left(\mathrm{M}\left(y_{n_{i}}, y_{m_{i}}, \mathrm{t}_{0}\right)\right)\right)\right\}\right)
\end{aligned}
$$

By (7), (8) and (9), letting $\mathrm{i} \rightarrow \infty$ in (9), we have

$$
\mathrm{g}\left(1-\in_{0}\right) \leq \phi\left(\max \left\{\mathrm{g}\left(1-\in_{0}\right), 0,0, \mathrm{~g}\left(1-\in_{0}\right)\right\}\right)
$$

$$
=\phi\left(\mathrm{g}\left(1-\in_{0}\right)\right)<\mathrm{g}\left(1-\in_{0}\right)
$$

which is a contradiction. Therefore, $\left\{\mathrm{y}_{\mathrm{n}}\right\}$ is a Cauchy-sequence in $\mathrm{X}$.

Now, we prove $\lim _{\mathrm{n} \rightarrow \infty} \mathrm{g}\left(\mathrm{M}\left(\mathrm{y}_{\mathrm{n}}, \mathrm{y}_{\mathrm{n}+1}, \mathrm{t}_{0}\right)\right)=0$ for all $t>0$. In fact, by (2) and (3), we have

$$
\begin{aligned}
& g\left(M\left(y_{2 n}, y_{2 n+1}, t\right)\right)= g\left(M\left(A x_{2 n}, B x_{2 n+1}, t\right)\right) \\
& \leq \phi\left(\operatorname { m a x } \left\{g\left(M\left(S_{2 n}, T x_{2 n+1}, t\right)\right),\right.\right. \\
& g\left(M\left(S_{2 n}, A x_{2 n}, t\right)\right), g\left(M\left(T_{2 n+1}, B x_{2 n+1}, t\right)\right), \\
&\left.\left.1 / 2\left(g\left(M\left(S_{2 n}, B x_{2 n+1}, t\right)\right)+g\left(M\left(T_{2 n+1}, A x_{2 n}, t\right)\right)\right)\right\}\right) \\
&= \phi\left(\operatorname { m a x } \left\{g\left(M\left(y_{2 n-1}, y_{2 n}, t\right)\right), g\left(M\left(y_{2 n-1}, y_{2 n}, t\right)\right),\right.\right. \\
&\left.\left.\left.g\left(M\left(y_{2 n}, y_{2 n+1}, t\right)\right), 1 / 2\left(g\left(M\left(y_{2 n-1}, y_{2 n+1}, t\right)\right)+g(1)\right)\right)\right\}\right) \\
& \leq \phi\left(\operatorname { m a x } \left\{g\left(M\left(y_{2 n-1}, y_{2 n}, t\right)\right), g\left(M\left(y_{2 n}, y_{2 n+1}, t\right)\right)\right.\right. \\
&\left.\left.1 / 2\left(g\left(M\left(y_{2 n-1}, y_{2 n}, t\right)\right)+g\left(M\left(y_{2 n}, y_{2 n+1}, t\right)\right)\right)\right\}\right) .
\end{aligned}
$$

If $\mathrm{g}\left(\mathrm{M}\left(\mathrm{y}_{2 \mathrm{n}-1}, \mathrm{y}_{2 \mathrm{n}}, \mathrm{t}\right)\right) \leq \mathrm{g}\left(\mathrm{M}\left(\mathrm{y}_{2 \mathrm{n}}, \mathrm{y}_{2 \mathrm{n}+1}, \mathrm{t}\right)\right)$ for all $\mathrm{t}>0$, then , by (2), $\mathrm{g}\left(\mathrm{M}\left(\mathrm{y}_{2 \mathrm{n}}, \mathrm{y}_{2 \mathrm{n}+1}, \mathrm{t}\right)\right) \leq \phi\left(\mathrm{g}\left(\mathrm{M}\left(\mathrm{y}_{2 \mathrm{n}}, \mathrm{y}_{2 \mathrm{n}+1}, \mathrm{t}\right)\right)\right)$ which means that , by Lemma 3.6, 
$\mathrm{g}\left(\mathrm{M}\left(\mathrm{y}_{2 \mathrm{n}}, \mathrm{y}_{2 \mathrm{n}+1}, \mathrm{t}\right)\right)=0$ for all $t>0$. Similarly, we have $\mathrm{g}\left(\mathrm{M}\left(\mathrm{y}_{2 \mathrm{n}+1}, \mathrm{y}_{2 \mathrm{n}+2}, \mathrm{t}\right)\right)=0$ for all $t>0$.

Thus we have $\lim _{\mathrm{n} \rightarrow \infty} \mathrm{g}\left(\mathrm{M}\left(\mathrm{y}_{\mathrm{n}} \mathrm{y}_{\mathrm{n}+1}, \mathrm{t}\right)\right)=0$ for all $t>0$.

On the other hand, if

$\mathrm{g}\left(\mathrm{M}\left(\mathrm{y}_{2 \mathrm{n}-1}, \mathrm{y}_{2 \mathrm{n}}, \mathrm{t}\right)\right) \geq \mathrm{g}\left(\mathrm{M}\left(\mathrm{y}_{2 \mathrm{n}}, \mathrm{y}_{2 \mathrm{n}+1}, \mathrm{t}\right)\right)$, then by $(2)$, we have

$\mathrm{g}\left(\mathrm{M}\left(\mathrm{y}_{2 \mathrm{n}}, \mathrm{y}_{2 \mathrm{n}+1}, \mathrm{t}\right)\right) \leq \phi\left(\mathrm{g}\left(\mathrm{M}\left(\mathrm{y}_{2 \mathrm{n}-1}, \mathrm{y}_{2 \mathrm{n}}, \mathrm{t}\right)\right)\right.$ for all $t>0$.

Similarly, $\mathrm{g}\left(\mathrm{M}\left(\mathrm{y}_{2 \mathrm{n}+1}, \mathrm{y}_{2 \mathrm{n}+2}, \mathrm{t}\right)\right) \leq \phi\left(\mathrm{g}\left(\mathrm{M}\left(\mathrm{y}_{2 \mathrm{n}}, \mathrm{y}_{2 \mathrm{n}+1}, \mathrm{t}\right)\right)\right)$, for all $t>0$. Thus we have

$\mathrm{g}\left(\mathrm{M}\left(\mathrm{y}_{\mathrm{n}}, \mathrm{y}_{\mathrm{n}+1}, \mathrm{t}\right)\right) \leq \phi\left(\mathrm{g}\left(\mathrm{M}\left(\mathrm{y}_{\mathrm{n}-1}, \mathrm{y}_{\mathrm{n}}, \mathrm{t}\right)\right)\right.$ for all $t>0$ and $n=1,2,3, \ldots$ Therefore by Lemma 3.6, $\lim _{\mathrm{n} \rightarrow \infty} \mathrm{g}\left(\mathrm{M}\left(\mathrm{y}_{\mathrm{n}}, \mathrm{y}_{\mathrm{n}+1}, \mathrm{t}\right)\right)=0$ for all $t>0$, which implies that $\left\{\mathrm{y}_{\mathrm{n}}\right\}$ is a Cauchy sequence in X by Lemma 3.7. Since (X, M, *) is complete, the sequence $\left\{\mathrm{y}_{\mathrm{n}}\right\}$ converges to a point $\mathrm{z} \in \mathrm{X}$ and so the subsequences $\left\{\mathrm{Ax}_{2 \mathrm{n}}\right\}$, $\left\{\mathrm{Bx}_{2 \mathrm{n}+1}\right\},\left\{\mathrm{Sx}_{2 \mathrm{n}}\right)$ and $\left\{\mathrm{Tx}_{2 \mathrm{n}+1}\right\}$ of $\left\{\mathrm{y}_{\mathrm{n}}\right\}$ also converges to the same limit $\mathrm{z}$, i.e., $\lim _{\mathrm{n} \rightarrow \infty} \mathrm{Ax}_{2 \mathrm{n}}=\lim _{\mathrm{n} \rightarrow \infty} \mathrm{Tx}_{2 \mathrm{n}+1}=\lim _{\mathrm{n} \rightarrow \infty} \mathrm{Bx}_{2 \mathrm{n}+1}=\lim _{\mathrm{n} \rightarrow \infty} \mathrm{Sx}_{2 \mathrm{n}+2}=\mathrm{z}$.

Case I ( $\mathrm{S}$ is continuous). In this case, we have

$\mathrm{SAx}_{2 \mathrm{n}} \rightarrow \mathrm{Sz}, \mathrm{S}^{2} \mathrm{x}_{2 \mathrm{n}} \rightarrow \mathrm{Sz}$.

The semi-compatibility of the pair $(\mathrm{A}, \mathrm{S})$ gives

$\lim$

$n \rightarrow \infty \mathrm{ASx}_{2 \mathrm{n}}=\mathrm{Sz}$.

Step I. By putting $\mathrm{x}=\mathrm{Sx}_{2 \mathrm{n}}, \mathrm{y}=\mathrm{x}_{2 \mathrm{n}+1}$ in (2), we obtain that $\mathrm{g}\left(\mathrm{M}\left(\mathrm{ASx}_{2 \mathrm{n}}, \mathrm{Bx}_{2 \mathrm{n}+1}, \mathrm{t}\right)\right) \leq \phi\left(\max \left\{\mathrm{g}\left(\mathrm{M}\left(\mathrm{SSx}_{2 \mathrm{n}}, \mathrm{Tx}_{2 \mathrm{n}+1}, \mathrm{t}\right)\right)\right.\right.$,

$$
\begin{aligned}
& \mathrm{g}\left(\mathrm{M}\left(\mathrm{SSx}_{2 \mathrm{n}}, \mathrm{ASx}_{2 \mathrm{n}}, \mathrm{t}\right)\right), \mathrm{g}\left(\mathrm{M}\left(\mathrm{Tx}_{2 \mathrm{n}+1}, \mathrm{Bx}_{2 \mathrm{n}+1}, \mathrm{t}\right)\right), \\
& \left.\left.\quad 1 / 2\left(\mathrm{~g}\left(\mathrm{M}\left(\mathrm{SSx}_{2 \mathrm{n}}, \mathrm{Bx_{2n+1 }}, \mathrm{t}\right)\right)+\mathrm{g}\left(\mathrm{M}\left(\mathrm{Tx}_{2 \mathrm{n}+1}, \mathrm{ASx}_{2 \mathrm{n}}, \mathrm{t}\right)\right)\right)\right\}\right)
\end{aligned}
$$

Letting $\mathrm{n} \rightarrow \infty$, we have

$\mathrm{g}(\mathrm{M}(\mathrm{Sz}, \mathrm{z}, \mathrm{t})) \leq \phi(\max \{\mathrm{g}(\mathrm{M}(\mathrm{Sz}, \mathrm{z}, \mathrm{t}))$,

$$
\begin{aligned}
& \mathrm{g}(\mathrm{M}(\mathrm{Sz}, \mathrm{Sz}, \mathrm{t})), \mathrm{g}(\mathrm{M}(\mathrm{z}, \mathrm{z}, \mathrm{t})), \\
& 1 / 2(\mathrm{~g}(\mathrm{M}(\mathrm{Sz}, \mathrm{z}, \mathrm{t}))+\mathrm{g}(\mathrm{M}(\mathrm{z}, \mathrm{Sz}, \mathrm{t})))\})
\end{aligned}
$$

$\Rightarrow \mathrm{g}(\mathrm{M}(\mathrm{Sz}, \mathrm{z}, \mathrm{t})) \leq \phi(\max \{\mathrm{g}(\mathrm{M}(\mathrm{Sz}, \mathrm{z}, \mathrm{t})), 0,0, \mathrm{~g}(\mathrm{M}(\mathrm{Sz}, \mathrm{z}, \mathrm{t})))\})$

$\Rightarrow \mathrm{g}(\mathrm{M}(\mathrm{Sz}, \mathrm{z}, \mathrm{t})) \leq \phi\{\mathrm{g}(\mathrm{M}(\mathrm{Sz}, \mathrm{z}, \mathrm{t})\}$

$\Rightarrow \mathrm{g}(\mathrm{M}(\mathrm{Sz}, \mathrm{z}, \mathrm{t}))=0$.i.e., $\mathrm{M}(\mathrm{Sz}, \mathrm{z}, \mathrm{t})=1$

$\Rightarrow \mathrm{z}=\mathrm{Sz}$.

Step II. By putting $\mathrm{x}=\mathrm{z}, \mathrm{y}=\mathrm{x}_{2 \mathrm{n}+1}$ in (2), we obtain that $\mathrm{g}\left(\mathrm{M}\left(\mathrm{Az}, \mathrm{Bx}_{2 \mathrm{n}+1}, \mathrm{t}\right)\right) \leq \phi\left(\max \left\{\mathrm{g}\left(\mathrm{M}\left(\mathrm{Sz}_{\mathrm{Tx}} \mathrm{Tx}_{2 \mathrm{n}+1}, \mathrm{t}\right)\right)\right.\right.$,

$$
\begin{aligned}
& \mathrm{g}(\mathrm{M}(\mathrm{Sz}, \mathrm{Az}, \mathrm{t})), \mathrm{g}\left(\mathrm{M}\left(\mathrm{Tx}_{2 \mathrm{n}+1}, \mathrm{Bx}_{2 \mathrm{n}+1}, \mathrm{t}\right)\right), \\
& \left.\left.\quad 1 / 2\left(\mathrm{~g}\left(\mathrm{M}\left(\mathrm{Sz}, \mathrm{Bx}_{2 \mathrm{n}+1}, \mathrm{t}\right)\right)+\mathrm{g}\left(\mathrm{M}\left(\mathrm{Tx}_{2 \mathrm{n}+1}, \mathrm{Az}, \mathrm{t}\right)\right)\right)\right\}\right)
\end{aligned}
$$

Letting $\mathrm{n} \rightarrow \infty$, we have 


$$
\begin{aligned}
& \mathrm{g}(\mathrm{M}(\mathrm{Az}, \mathrm{z}, \mathrm{t})) \leq \phi(\max \{\mathrm{g}(\mathrm{M}(\mathrm{z}, \mathrm{z}, \mathrm{t})), \\
& \mathrm{g}(\mathrm{M}(\mathrm{z}, \mathrm{Az}, \mathrm{t})), \mathrm{g}(\mathrm{M}(\mathrm{z}, \mathrm{z}, \mathrm{t})) \text {, } \\
& 1 / 2(\mathrm{~g}(\mathrm{M}(\mathrm{z}, \mathrm{z}, \mathrm{t}))+\mathrm{g}(\mathrm{M}(\mathrm{z}, \mathrm{Az}, \mathrm{t})))\}) \\
& \Rightarrow \mathrm{g}(\mathrm{M}(\mathrm{Az}, \mathrm{z}, \mathrm{t})) \leq \phi(\max \{0, \mathrm{~g}(\mathrm{M}(\mathrm{z}, \mathrm{Az}, \mathrm{t})), 0,1 / 2(\mathrm{~g}(\mathrm{M}(\mathrm{z}, \mathrm{Az}, \mathrm{t})))\}) \\
& \Rightarrow \mathrm{g}(\mathrm{M}(\mathrm{Az}, \mathrm{z}, \mathrm{t})) \leq \phi\{\mathrm{g}(\mathrm{M}(\mathrm{Az}, \mathrm{z}, \mathrm{t})\} \\
& \Rightarrow \mathrm{g}(\mathrm{M}(\mathrm{Az}, \mathrm{z}, \mathrm{t}))=0 \text {.i.e., } \mathrm{M}(\mathrm{Az}, \mathrm{z}, \mathrm{t})=1 \\
& \Rightarrow \mathrm{z}=\mathrm{Az} \text {. }
\end{aligned}
$$

Hence, $\quad \mathrm{Az}=\mathrm{z}=\mathrm{Sz}$.

Step III. As $\mathrm{A}(\mathrm{X}) \subset \mathrm{T}(\mathrm{X})$, there exists a point $\mathrm{w} \in \mathrm{X}$ such that $\mathrm{Az}=\mathrm{Sz}=\mathrm{z}=\mathrm{Tw}$. By putting $\mathrm{x}=\mathrm{x}_{2 \mathrm{n}}, \mathrm{y}=\mathrm{w}$ in (2), we obtain that $\mathrm{g}\left(\mathrm{M}\left(\mathrm{Ax}_{2 \mathrm{n}}, \mathrm{Bw}, \mathrm{t}\right)\right) \leq \phi\left(\max \left\{\mathrm{g}\left(\mathrm{M}\left(\mathrm{Sx}_{2 \mathrm{n}}, \mathrm{Tw}, \mathrm{t}\right)\right)\right.\right.$,

$$
\begin{aligned}
& \mathrm{g}\left(\mathrm{M}\left(\mathrm{Sx}_{2 \mathrm{n}}, \mathrm{Ax} \mathrm{x}_{2 \mathrm{n}}, \mathrm{t}\right)\right), \mathrm{g}(\mathrm{M}(\mathrm{Tw}, \mathrm{Bw}, \mathrm{t})), \\
& \left.\left.\quad 1 / 2\left(\mathrm{~g}\left(\mathrm{M}\left(\mathrm{Sx}_{2 \mathrm{n}}, \mathrm{Bw}, \mathrm{t}\right)\right)+\mathrm{g}\left(\mathrm{M}\left(\mathrm{Tw}, \mathrm{Ax}_{2 \mathrm{n}}, \mathrm{t}\right)\right)\right)\right\}\right)
\end{aligned}
$$

Letting $\mathrm{n} \rightarrow \infty$, we have

$$
\begin{aligned}
& \mathrm{g}(\mathrm{M}(\mathrm{z}, \mathrm{Bw}, \mathrm{t})) \leq \phi(\max \{\mathrm{g}(\mathrm{M}(\mathrm{z}, \mathrm{z}, \mathrm{t})), \\
& \mathrm{g}(\mathrm{M}(\mathrm{z}, \mathrm{z}, \mathrm{t})), \mathrm{g}(\mathrm{M}(\mathrm{z}, \mathrm{Bw}, \mathrm{t})), \\
& 1 / 2(\mathrm{~g}(\mathrm{M}(\mathrm{z}, \mathrm{Bw}, \mathrm{t}))+\mathrm{g}(\mathrm{M}(\mathrm{z}, \mathrm{z}, \mathrm{t})))\}) \\
& \mathrm{g}(\mathrm{M}(\mathrm{z}, \mathrm{Bw}, \mathrm{t})) \leq \phi(\max \{0,0, \mathrm{~g}(\mathrm{M}(\mathrm{z}, \mathrm{Bw}, \mathrm{t})), 1 / 2(\mathrm{~g}(\mathrm{M}(\mathrm{z}, \mathrm{Bw}, \mathrm{t})))\}) \\
& \Rightarrow \mathrm{g}(\mathrm{M}(\mathrm{z}, \mathrm{Bw}, \mathrm{t})) \leq \phi(\mathrm{g}(\mathrm{M}(\mathrm{z}, \mathrm{Bw}, \mathrm{t})) \\
& \Rightarrow \mathrm{g}(\mathrm{M}(\mathrm{z}, \mathrm{Bw}, \mathrm{t}))=0 \text {.i.e., } \mathrm{M}(\mathrm{z}, \mathrm{Bw}, \mathrm{t})=1 \\
& \Rightarrow \mathrm{z}=\mathrm{Bw} .
\end{aligned}
$$

Therefore $\mathrm{Bw}=\mathrm{Tw}=\mathrm{z}$. Since $(\mathrm{B}, \mathrm{T})$ is weakly compatible, we get that $\mathrm{TBw}=\mathrm{BTw}$, that is, $\mathrm{Bz}=\mathrm{Tz}$.

Step IV. By putting $\mathrm{x}=\mathrm{z}, \mathrm{y}=\mathrm{z}$ in (2), we obtain that $\mathrm{g}(\mathrm{M}(\mathrm{Az}, \mathrm{Bz}, \mathrm{t})) \leq \phi(\max \{\mathrm{g}(\mathrm{M}(\mathrm{Sz}, \mathrm{Tz}, \mathrm{t}))$

$$
\begin{aligned}
& \mathrm{g}(\mathrm{M}(\mathrm{Sz}, \mathrm{Az}, \mathrm{t})), \mathrm{g}(\mathrm{M}(\mathrm{Tz}, \mathrm{Bz}, \mathrm{t})), \\
& \quad 1 / 2(\mathrm{~g}(\mathrm{M}(\mathrm{Sz}, \mathrm{Bz}, \mathrm{t}))+\mathrm{g}(\mathrm{M}(\mathrm{Tz}, \mathrm{Az}, \mathrm{t})))\})
\end{aligned}
$$

$\mathrm{g}(\mathrm{M}(\mathrm{Az}, \mathrm{Bz}, \mathrm{t})) \leq \phi(\max \{\mathrm{g}(\mathrm{M}(\mathrm{Az}, \mathrm{Bz}, \mathrm{t}))$,

$$
\begin{aligned}
& \mathrm{g}(\mathrm{M}(\mathrm{z}, \mathrm{z}, \mathrm{t})), \mathrm{g}(\mathrm{M}(\mathrm{Bz}, \mathrm{Bz}, \mathrm{t})), \\
& \quad 1 / 2(\mathrm{~g}(\mathrm{M}(\mathrm{Az}, \mathrm{Bz}, \mathrm{t}))+\mathrm{g}(\mathrm{M}(\mathrm{Bz}, \mathrm{Az}, \mathrm{t})))\})
\end{aligned}
$$

$\mathrm{g}(\mathrm{M}(\mathrm{Az}, \mathrm{Bz}, \mathrm{t})) \leq \phi(\max \{\mathrm{g}(\mathrm{M}(\mathrm{Az}, \mathrm{Bz}, \mathrm{t})), 0,0, \mathrm{~g}(\mathrm{M}(\mathrm{Az}, \mathrm{Bz}, \mathrm{t}))\})$

$\Rightarrow \mathrm{g}(\mathrm{M}(\mathrm{Az}, \mathrm{Bz}, \mathrm{t})) \leq \phi(\mathrm{g}(\mathrm{M}(\mathrm{Az}, \mathrm{Bz}, \mathrm{t}))$ 
$\Rightarrow \mathrm{g}(\mathrm{M}(\mathrm{Az}, \mathrm{Bz}, \mathrm{t}))=0$.i.e., $\mathrm{M}(\mathrm{Az}, \mathrm{Bz}, \mathrm{t})=1$

$\Rightarrow \mathrm{Az}=\mathrm{Bz}$.

Therefore, $\mathrm{z}=\mathrm{Az}=\mathrm{Sz}=\mathrm{Bz}=\mathrm{Tz}$, that is, $\mathrm{z}$ is a common fixed point of $\mathrm{A}, \mathrm{B}$, $\mathrm{S}$ and $\mathrm{T}$.

Case II (A is continuous). In this case, we have $\mathrm{ASx}_{2 \mathrm{n}} \rightarrow \mathrm{Az}$

The semi-compatibility of the pair (A, S) gives $\mathrm{ASx}_{2 \mathrm{n}} \rightarrow \mathrm{Sz}$.

By uniqueness of limit in N.A. FM Space, we obtain that $\mathrm{Az}=\mathrm{Sz}$.

Step $\boldsymbol{V}$. By putting $\mathrm{x}=\mathrm{z}, \mathrm{y}=\mathrm{x}_{2 \mathrm{n}+1}$ in (2), we obtain that $\mathrm{g}\left(\mathrm{M}\left(\mathrm{Az}, \mathrm{Bx}_{2 \mathrm{n}+1}, \mathrm{t}\right)\right) \leq \phi\left(\max \left\{\mathrm{g}\left(\mathrm{M}\left(\mathrm{Sz}, \mathrm{Tx}_{2 \mathrm{n}+1}, \mathrm{t}\right)\right)\right.\right.$,

$$
\begin{aligned}
& \mathrm{g}(\mathrm{M}(\mathrm{Sz}, \mathrm{Az}, \mathrm{t})), \mathrm{g}\left(\mathrm{M}\left(\mathrm{Tx}_{2 \mathrm{n}+1}, \mathrm{Bx} \mathrm{x}_{2 \mathrm{n}+1}, \mathrm{t}\right)\right), \\
& \left.\left.\quad 1 / 2\left(\mathrm{~g}\left(\mathrm{M}\left(\mathrm{Sz}, \mathrm{Bx}_{2 \mathrm{n}+1}, \mathrm{t}\right)\right)+\mathrm{g}\left(\mathrm{M}\left(\mathrm{Tx}_{2 \mathrm{n}+1}, \mathrm{Az}, \mathrm{t}\right)\right)\right)\right\}\right)
\end{aligned}
$$

Letting $\mathrm{n} \rightarrow \infty$, we have

$$
\begin{gathered}
\mathrm{g}(\mathrm{M}(\mathrm{Az}, \mathrm{z}, \mathrm{t})) \leq \phi(\max \{\mathrm{g}(\mathrm{M}(\mathrm{z}, \mathrm{z}, \mathrm{t})), \\
\mathrm{g}(\mathrm{M}(\mathrm{z}, \mathrm{Az}, \mathrm{t})), \mathrm{g}(\mathrm{M}(\mathrm{z}, \mathrm{z}, \mathrm{t})), \\
1 / 2(\mathrm{~g}(\mathrm{M}(\mathrm{z}, \mathrm{z}, \mathrm{t}))+\mathrm{g}(\mathrm{M}(\mathrm{z}, \mathrm{Az}, \mathrm{t})))\}) \\
\Rightarrow \mathrm{g}(\mathrm{M}(\mathrm{Az}, \mathrm{z}, \mathrm{t})) \leq \phi(\max \{0, \mathrm{~g}(\mathrm{M}(\mathrm{z}, \mathrm{Az}, \mathrm{t})), 0,1 / 2(\mathrm{~g}(\mathrm{M}(\mathrm{z}, \mathrm{Az}, \mathrm{t})))\}) \\
\Rightarrow \mathrm{g}(\mathrm{M}(\mathrm{Az}, \mathrm{z}, \mathrm{t})) \leq \phi\{\mathrm{g}(\mathrm{M}(\mathrm{Az}, \mathrm{z}, \mathrm{t})\} \\
\Rightarrow \mathrm{g}(\mathrm{M}(\mathrm{Az}, \mathrm{z}, \mathrm{t}))=0 \text { i.e., } \mathrm{M}(\mathrm{Az}, \mathrm{z}, \mathrm{t})=1
\end{gathered}
$$

$\Rightarrow \mathrm{z}=\mathrm{Az}$ and rest of the proof follows from Step III onwards of the previous case.

Uniqueness. Let $u$ be another common fixed point of $\mathrm{A}, \mathrm{B}, \mathrm{S}$ and $\mathrm{T}$.

Then $\mathrm{u}=\mathrm{Au}=\mathrm{Su}=\mathrm{Bu}=\mathrm{Tu}$.

Putting $\mathrm{x}=\mathrm{z}$ and $\mathrm{y}=\mathrm{u}$ in (2.2), we have

$\mathrm{g}(\mathrm{M}(\mathrm{Az}, \mathrm{Bu}, \mathrm{t})) \leq \phi(\max \{\mathrm{g}(\mathrm{M}(\mathrm{Sz}, \mathrm{Tu}, \mathrm{t})), \mathrm{g}(\mathrm{M}(\mathrm{Sz}, \mathrm{Az}, \mathrm{t})), \mathrm{g}(\mathrm{M}(\mathrm{Tu}, \mathrm{Bu}, \mathrm{t}))$,

$$
1 / 2(\mathrm{~g}(\mathrm{M}(\mathrm{Sz}, \mathrm{Bu}, \mathrm{t}))+\mathrm{g}(\mathrm{M}(\mathrm{Tu}, \mathrm{Az}, \mathrm{t})))\}),
$$

$\Rightarrow \mathrm{g}(\mathrm{M}(\mathrm{z}, \mathrm{u}, \mathrm{t})) \leq \phi(\max \{\mathrm{g}(\mathrm{M}(\mathrm{z}, \mathrm{u}, \mathrm{t})), \mathrm{g}(\mathrm{M}(\mathrm{z}, \mathrm{z}, \mathrm{t})), \mathrm{g}(\mathrm{M}(\mathrm{u}, \mathrm{u}, \mathrm{t}))$,

$$
1 / 2(\mathrm{~g}(\mathrm{M}(\mathrm{z}, \mathrm{u}, \mathrm{t}))+\mathrm{g}(\mathrm{M}(\mathrm{u}, \mathrm{z}, \mathrm{t})))\})
$$

$\Rightarrow \mathrm{g}(\mathrm{M}(\mathrm{z}, \mathrm{u}, \mathrm{t})) \leq \phi(\max \{\mathrm{g}(\mathrm{M}(\mathrm{z}, \mathrm{u}, \mathrm{t})), 0,0, \mathrm{~g}(\mathrm{M}(\mathrm{z}, \mathrm{u}, \mathrm{t}))\})$,

$\Rightarrow \mathrm{g}(\mathrm{M}(\mathrm{z}, \mathrm{u}, \mathrm{t})) \leq \phi\{\mathrm{g}(\mathrm{M}(\mathrm{z}, \mathrm{u}, \mathrm{t})\}$

$\Rightarrow \mathrm{g}(\mathrm{M}(\mathrm{z}, \mathrm{u}, \mathrm{t}))=0$.i.e., $\mathrm{M}(\mathrm{z}, \mathrm{u}, \mathrm{t})=1$

$\Rightarrow \mathrm{z}=\mathrm{u}$.

Therefore, $\mathrm{z}$ is the unique common fixed point of the self-maps $\mathrm{A}, \mathrm{B}, \mathrm{S}$ and 
$\mathrm{T}$.

Corollary 4.2. Let $\mathrm{A}, \mathrm{B}, \mathrm{S}, \mathrm{T}: \mathrm{X} \rightarrow \mathrm{X}$ be mappings such that

(4.2.1) $\mathrm{A}(\mathrm{X}) \subset \mathrm{T}(\mathrm{X})$ and $\mathrm{B}(\mathrm{X}) \subset \mathrm{S}(\mathrm{X})$,

(4.2.2) $\mathrm{g}(\mathrm{M}(\mathrm{Ax}, \mathrm{By}, \mathrm{t})) \leq \phi(\max \{\mathrm{g}(\mathrm{M}(\mathrm{Sx}, \mathrm{Ty}, \mathrm{t})), \mathrm{g}(\mathrm{M}(\mathrm{Sx}, \mathrm{Ax}, \mathrm{t})), \mathrm{g}(\mathrm{M}(\mathrm{Ty}, \mathrm{By}, \mathrm{t}))$,

$1 / 2(\mathrm{~g}(\mathrm{M}(\mathrm{Sx}, \mathrm{By}, \mathrm{t}))+\mathrm{g}(\mathrm{M}(\mathrm{Ty}, \mathrm{Ax}, \mathrm{t})))\})$ for all $\mathrm{t}>0$,

where a function $\phi:[0, \infty) \rightarrow[0, \infty)$ satisfies the condition $(\Phi)$.

(4.2.3) the pairs $(\mathrm{A}, \mathrm{S})$ and $(\mathrm{B}, \mathrm{T})$ are semicompatible.

(4.2.4) one of $\mathrm{A}, \mathrm{B}, \mathrm{S}$ or $\mathrm{T}$ is continuous

Then A, B,S and Thave a unique common fixed point in $\mathrm{X}$.

Proof. As semi-compatibility implies weak compatibility, the proof follows from theorem 4.1.

On taking A = B in theorem 4.1, we have the following corollary.

Corollary 4.3. Let $\mathrm{A}, \mathrm{S}$ and $\mathrm{T}: \mathrm{X} \rightarrow \mathrm{X}$ be mappings such that

(4.3.1) $\mathrm{A}(\mathrm{X}) \subset \mathrm{T}(\mathrm{X}) \cap \mathrm{S}(\mathrm{X})$,

(4.3.2) $\mathrm{g}(\mathrm{M}(\mathrm{Ax}, \mathrm{Ay}, \mathrm{t})) \leq \phi(\max \{\mathrm{g}(\mathrm{M}(\mathrm{Sx}, \mathrm{Ty}, \mathrm{t})), \mathrm{g}(\mathrm{M}(\mathrm{Sx}, \mathrm{Ax}, \mathrm{t})), \mathrm{g}(\mathrm{M}(\mathrm{Ty}, \mathrm{Ay}, \mathrm{t}))$,

$1 / 2(\mathrm{~g}(\mathrm{M}(\mathrm{Sx}, \mathrm{Ay}, \mathrm{t}))+\mathrm{g}(\mathrm{M}(\mathrm{Ty}, \mathrm{Ax}, \mathrm{t})))\})$ for all $\mathrm{t}>0$,

where a function $\phi:[0, \infty) \rightarrow[0, \infty)$ satisfies the condition $(\Phi)$.

(4.3.3) the pair $(\mathrm{A}, \mathrm{S})$ is semi-compatible and $(\mathrm{A}, \mathrm{T})$ is weakly compatible

(4.3.4) one of $\mathrm{A}$ or $\mathrm{S}$ is continuous

Then $\mathrm{A}, \mathrm{S}$ and $\mathrm{T}$ have a unique common fixed point in $\mathrm{X}$.

Now, taking $\mathrm{S}=\mathrm{I}$ and $\mathrm{T}=\mathrm{I}$ in theorem in 4.1, the conditions (1),(3) and (4) are satisfied trivially, and we get the following corollary.

Corollary 4.4. Let $\mathrm{A}, \mathrm{B}: \mathrm{X} \rightarrow \mathrm{X}$ be mappings such that

(4.4.1) $\mathrm{g}(\mathrm{M}(\mathrm{Ax}, \mathrm{By}, \mathrm{t})) \leq \phi(\max \{\mathrm{g}(\mathrm{M}(\mathrm{x}, \mathrm{y}, \mathrm{t})), \mathrm{g}(\mathrm{M}(\mathrm{x}, \mathrm{Ax}, \mathrm{t}))$, $\mathrm{g}(\mathrm{M}(\mathrm{y}, \mathrm{By}, \mathrm{t})), 1 / 2(\mathrm{~g}(\mathrm{M}(\mathrm{x}, \mathrm{By}, \mathrm{t}))+\mathrm{g}(\mathrm{M}(\mathrm{y}, \mathrm{Ax}, \mathrm{t})))\})$ for all $\mathrm{t}>0$,

where a function $\phi:[0, \infty) \rightarrow[0, \infty)$ satisfies the condition $(\Phi)$.

Then A, B have a unique common fixed point in X.

Theorem 4.5. Let $\mathrm{A}, \mathrm{B}, \mathrm{S}, \mathrm{T}: \mathrm{X} \rightarrow \mathrm{X}$ be mappings satisfying (1), (2) and the following :

(4.5.1) the pair $(\mathrm{A}, \mathrm{S})$ is compatible and $(\mathrm{B}, \mathrm{T})$ is weakly compatible

(4.5.2) one of $\mathrm{A}$ or $\mathrm{S}$ is continuous

Then A, B, S and T have a unique common fixed point in $\mathrm{X}$.

Proof. In view of Proposition (3.1) and theorem (4.1), it suffices to prove the result when $A$ is continuous. As in the proof of theorem 4.1, the sequence $\left\{\mathrm{y}_{\mathrm{n}}\right\}$ $\rightarrow \mathrm{z} \in \mathrm{X}$ and (a) are satisfied. As $A$ is continuous, we have $\mathrm{ASx}_{2 \mathrm{n}} \rightarrow \mathrm{Az}, \mathrm{AAx}_{2 \mathrm{n}} \rightarrow \mathrm{Az}$.

The compatibility of $(\mathrm{A}, \mathrm{S})$ gives $\lim _{n \rightarrow \infty} \mathrm{ASx}_{2 \mathrm{n}}=\mathrm{Az}=\lim _{n \rightarrow \infty} \mathrm{SAx}_{2 \mathrm{n}}$.

Step I. By putting $\mathrm{x}=\mathrm{Ax}_{2 \mathrm{n}}, \mathrm{y}=\mathrm{x}_{2 \mathrm{n}+1}$ in (2), we obtain that $\mathrm{g}\left(\mathrm{M}\left(\mathrm{AAx}_{2 \mathrm{n}}, \mathrm{Bx}_{2 \mathrm{n}+1}, \mathrm{t}\right)\right) \leq \phi\left(\max \left\{\mathrm{g}\left(\mathrm{M}\left(\mathrm{SAx}_{2 \mathrm{n}}, \mathrm{Tx}_{2 \mathrm{n}+1}, \mathrm{t}\right)\right)\right.\right.$,

$$
\mathrm{g}\left(\mathrm{M}\left(\mathrm{SAx}_{2 \mathrm{n}}, \mathrm{AAx}_{2 \mathrm{n}}, \mathrm{t}\right)\right), \mathrm{g}\left(\mathrm{M}\left(\mathrm{Tx}_{2 \mathrm{n}+1}, \mathrm{Bx}_{2 \mathrm{n}+1}, \mathrm{t}\right)\right),
$$




$$
\left.\left.1 / 2\left(\mathrm{~g}\left(\mathrm{M}\left(\mathrm{SAx}_{2 \mathrm{n}}, \mathrm{Bx}_{2 \mathrm{n}+1}, \mathrm{t}\right)\right)+\mathrm{g}\left(\mathrm{M}\left(\mathrm{Tx}_{2 \mathrm{n}+1}, \mathrm{AAx}_{2 \mathrm{n}}, \mathrm{t}\right)\right)\right)\right\}\right)
$$

Letting $n \rightarrow \infty$, using (a) we have

$$
\begin{aligned}
& \mathrm{g}(\mathrm{M}(\mathrm{Az}, \mathrm{z}, \mathrm{t})) \leq \phi(\max \{\mathrm{g}(\mathrm{M}(\mathrm{Az}, \mathrm{z}, \mathrm{t})), \mathrm{g}(\mathrm{M}(\mathrm{Az}, \mathrm{Az}, \mathrm{t})), \mathrm{g}(\mathrm{M}(\mathrm{z}, \mathrm{z}, \mathrm{t})) \\
& 1 / 2(\mathrm{~g}(\mathrm{M}(\mathrm{Az}, \mathrm{z}, \mathrm{t}))+\mathrm{g}(\mathrm{M}(\mathrm{z}, \mathrm{Az}, \mathrm{t})))\}) \\
& \Rightarrow \mathrm{g}(\mathrm{M}(\mathrm{Az}, \mathrm{z}, \mathrm{t})) \leq \phi(\max \{\mathrm{g}(\mathrm{M}(\mathrm{Az}, \mathrm{z}, \mathrm{t})), 0,0, \mathrm{~g}(\mathrm{M}(\mathrm{Az}, \mathrm{z}, \mathrm{t})))\}) \\
& \Rightarrow \mathrm{g}(\mathrm{M}(\mathrm{Az}, \mathrm{z}, \mathrm{t})) \leq \phi\{\mathrm{g}(\mathrm{M}(\mathrm{Az}, \mathrm{z}, \mathrm{t})\} \\
& \Rightarrow \mathrm{g}(\mathrm{M}(\mathrm{Az}, \mathrm{z}, \mathrm{t}))=0 \text {.i.e., } \mathrm{M}(\mathrm{Az}, \mathrm{z}, \mathrm{t})=1 \\
& \Rightarrow \mathrm{z}=\mathrm{Az}
\end{aligned}
$$

Step II. As $\mathrm{A}(\mathrm{X}) \subset \mathrm{T}(\mathrm{X})$, there exists a point $\mathrm{w} \in \mathrm{X}$ such that $\mathrm{Az}=\mathrm{Sz}=\mathrm{z}=\mathrm{Tw}$. By putting $\mathrm{x}=\mathrm{x}_{2 \mathrm{n}}, \mathrm{y}=\mathrm{w}$ in (2), we obtain that $\mathrm{g}\left(\mathrm{M}\left(\mathrm{Ax}_{2 \mathrm{n}}, \mathrm{Bw}, \mathrm{t}\right)\right) \leq \phi\left(\max \left\{\mathrm{g}\left(\mathrm{M}\left(\mathrm{Sx}_{2 \mathrm{n}}, \mathrm{Tw}, \mathrm{t}\right)\right), \mathrm{g}\left(\mathrm{M}\left(\mathrm{Sx}_{2 \mathrm{n}}, \mathrm{Ax} x_{2 \mathrm{n}}, \mathrm{t}\right)\right)\right.\right.$,

$$
\left.\left.\mathrm{g}(\mathrm{M}(\mathrm{Tw}, \mathrm{Bw}, \mathrm{t})), 1 / 2\left(\mathrm{~g}\left(\mathrm{M}\left(\mathrm{Sx}_{2 \mathrm{n}}, \mathrm{Bw}, \mathrm{t}\right)\right)+\mathrm{g}\left(\mathrm{M}\left(\mathrm{Tw}, \mathrm{Ax}_{2 \mathrm{n}}, \mathrm{t}\right)\right)\right)\right\}\right)
$$

Letting $\mathrm{n} \rightarrow \infty$, using (a), and the continuity of the t-norm, we have $\mathrm{g}(\mathrm{M}(\mathrm{z}, \mathrm{Bw}, \mathrm{t})) \leq \phi(\max \{\mathrm{g}(\mathrm{M}(\mathrm{z}, \mathrm{z}, \mathrm{t})), \mathrm{g}(\mathrm{M}(\mathrm{z}, \mathrm{z}, \mathrm{t})), \mathrm{g}(\mathrm{M}(\mathrm{z}, \mathrm{Bw}, \mathrm{t}))$,

$$
1 / 2(\mathrm{~g}(\mathrm{M}(\mathrm{z}, \mathrm{Bw}, \mathrm{t}))+\mathrm{g}(\mathrm{M}(\mathrm{z}, \mathrm{z}, \mathrm{t})))\})
$$

$\mathrm{g}(\mathrm{M}(\mathrm{z}, \mathrm{Bw}, \mathrm{t})) \leq \phi(\max \{0,0, \mathrm{~g}(\mathrm{M}(\mathrm{z}, \mathrm{Bw}, \mathrm{t})), 1 / 2(\mathrm{~g}(\mathrm{M}(\mathrm{z}, \mathrm{Bw}, \mathrm{t})))\})$

$\Rightarrow \mathrm{g}(\mathrm{M}(\mathrm{z}, \mathrm{Bw}, \mathrm{t})) \leq \phi(\mathrm{g}(\mathrm{M}(\mathrm{z}, \mathrm{Bw}, \mathrm{t}))$

$\Rightarrow \mathrm{g}(\mathrm{M}(\mathrm{z}, \mathrm{Bw}, \mathrm{t}))=0$.i.e., $\mathrm{M}(\mathrm{z}, \mathrm{Bw}, \mathrm{t})=1$

$\Rightarrow \mathrm{z}=\mathrm{Bw}$.

Therefore $\mathrm{Bw}=\mathrm{Tw}=\mathrm{z}$. Since $(\mathrm{B}, \mathrm{T})$ is weakly compatible, we get that $\mathrm{TBw}=\mathrm{BTw}$, that is, $\mathrm{Bz}=\mathrm{Tz}$.

Step III. Again as $\mathrm{z}=\mathrm{Bw}$ and $\mathrm{B}(\mathrm{X}) \subset \mathrm{S}(\mathrm{X})$, there exists $\mathrm{v} \in \mathrm{X}$ such that $\mathrm{z}=$ $\mathrm{Bz}=\mathrm{Sv}$. By putting $\mathrm{x}=\mathrm{v}, \mathrm{y}=\mathrm{w}$ in (2), we have $\mathrm{g}(\mathrm{M}(\mathrm{Av}, \mathrm{Bw}, \mathrm{t})) \leq \phi(\max \{\mathrm{g}(\mathrm{M}(\mathrm{Sv}, \mathrm{Tw}, \mathrm{t})), \mathrm{g}(\mathrm{M}(\mathrm{Sv}, \mathrm{Av}, \mathrm{t})), \mathrm{g}(\mathrm{M}(\mathrm{Tw}, \mathrm{Bw}, \mathrm{t}))$,

$1 / 2(\mathrm{~g}(\mathrm{M}(\mathrm{Sv}, \mathrm{Bw}, \mathrm{t}))+\mathrm{g}(\mathrm{M}(\mathrm{Tw}, \mathrm{Av}, \mathrm{t})))\})$,

$\Rightarrow \mathrm{g}(\mathrm{M}(\mathrm{Av}, \mathrm{Sv}, \mathrm{t})) \leq \phi(\max \{\mathrm{g}(\mathrm{M}(\mathrm{Sv}, \mathrm{Tw}, \mathrm{t})), \mathrm{g}(\mathrm{M}(\mathrm{Sv}, \mathrm{Av}, \mathrm{t})), \mathrm{g}(\mathrm{M}(\mathrm{Tw}, \mathrm{Sv}, \mathrm{t}))$, $1 / 2(\mathrm{~g}(\mathrm{M}(\mathrm{Sv}, \mathrm{Sv}, \mathrm{t}))+\mathrm{g}(\mathrm{M}(\mathrm{Tw}, \mathrm{Av}, \mathrm{t})))\})$,

$\Rightarrow \mathrm{g}(\mathrm{M}(\mathrm{Av}, \mathrm{Sv}, \mathrm{t})) \leq \phi(\max \{0, \mathrm{~g}(\mathrm{M}(\mathrm{Sv}, \mathrm{Av}, \mathrm{t})), 0,1 / 2(\mathrm{~g}(\mathrm{M}(\mathrm{Sv}, \mathrm{Av}, \mathrm{t})))\})$,

$\Rightarrow \mathrm{g}(\mathrm{M}(\mathrm{Av}, \mathrm{Sv}, \mathrm{t})) \leq \phi(\mathrm{g}(\mathrm{M}(\mathrm{Sv}, \mathrm{Av}, \mathrm{t}))$

$\Rightarrow \mathrm{g}(\mathrm{M}(\mathrm{Av}, \mathrm{Sv}, \mathrm{t}))=0$, that is, $\mathrm{M}(\mathrm{Av}, \mathrm{Sv}, \mathrm{t})=1$

$\Rightarrow \mathrm{Av}=\mathrm{Sv}$. As $(\mathrm{A}, \mathrm{S})$ is compatible, we have $\mathrm{ASv}=\mathrm{SAv}$ or $\mathrm{Az}=\mathrm{Sz}=\mathrm{z}$. 
Also $\mathrm{Az}=\mathrm{Bz}$ follows from Step IV in the proof of Theorem 4.1 and it follows that $\mathrm{z}$ is a common fixed point of four maps $\mathrm{A}, \mathrm{B}, \mathrm{S}$ and $\mathrm{T}$. The uniqueness follows as in the proof of theorem 4.1.

Corollary 4.6. Let $\mathrm{A}, \mathrm{B}, \mathrm{S}, \mathrm{T}: \mathrm{X} \rightarrow \mathrm{X}$ be mappings satisfying (1), (2) and the following :

(4.6.1) the pairs $(\mathrm{A}, \mathrm{S})$ and $(\mathrm{B}, \mathrm{T})$ are compatible,

(4.6.2) one of $\mathrm{A}, \mathrm{B}, \mathrm{S}$ or $\mathrm{T}$ is continuous

Then $\mathrm{A}, \mathrm{B}, \mathrm{S}$ and $\mathrm{T}$ have a unique common fixed point in $\mathrm{X}$.

Proof. As compatibility implies weak compatibility, the proof follows from theorem (4.5)

If we take $\mathrm{A}=\mathrm{I}$, the identity map on $X$ in Theorem (4.5), we have the following result for three self-maps, none of which is continuous and just a pair of them is needed to be weak compatible.

\section{Application}

Theorem 5.1. Let $(\mathrm{X}, \mathrm{M}, *)$ be a complete N.A. FM-space and $\mathrm{A}, \mathrm{B}, \mathrm{S}$ and $\mathrm{T}$ be the mappings from the product $\mathrm{X} \times \mathrm{X}$ to $\mathrm{X}$ such that

(5.1.1) $\mathrm{A}(\mathrm{X} \times\{\mathrm{y}\}) \subset \mathrm{T}(\mathrm{X} \times\{\mathrm{y}\})$ and $\mathrm{B}(\mathrm{X} \times\{\mathrm{y}\}) \subset(\mathrm{X} \times\{\mathrm{y}\})$

(5.1.2) $\mathrm{g}(\mathrm{M}(\mathrm{A}(\mathrm{T}(\mathrm{x}, \mathrm{y}), \mathrm{y}), \mathrm{T}(\mathrm{A}(\mathrm{x}, \mathrm{y}), \mathrm{y}), \mathrm{t})) \leq \mathrm{g}(\mathrm{M}(\mathrm{A}(\mathrm{x}, \mathrm{y}), \mathrm{T}(\mathrm{x}, \mathrm{y}), \mathrm{t}))$, $\mathrm{g}(\mathrm{M}(\mathrm{B}(\mathrm{S}(\mathrm{x}, \mathrm{y}), \mathrm{y}), \mathrm{S}(\mathrm{B}(\mathrm{x}, \mathrm{y}), \mathrm{y}), \mathrm{t})) \leq \mathrm{g}(\mathrm{M}(\mathrm{B}(\mathrm{x}, \mathrm{y}), \mathrm{S}(\mathrm{x}, \mathrm{y}), \mathrm{t}))$ for all $\mathrm{t}>0$.

$\mathrm{g}\left(\mathrm{M}\left(\mathrm{A}(\mathrm{x}, \mathrm{y}), \mathrm{B}\left(\mathrm{x}^{\prime}, \mathrm{y}^{\prime}\right), \mathrm{t}\right)\right) \leq \phi\left(\max \left\{\mathrm{g}\left(\mathrm{M}\left(\mathrm{S}(\mathrm{x}, \mathrm{y}), \mathrm{T}\left(\mathrm{x}^{\prime}, \mathrm{y}^{\prime}\right), \mathrm{t}\right)\right), \mathrm{M}(\mathrm{S}(\mathrm{x}, \mathrm{y}), \mathrm{A}(\mathrm{x}, \mathrm{y}), \mathrm{t})\right)\right.$, $\left.\left.\mathrm{g}\left(\mathrm{M}\left(\mathrm{T}\left(\mathrm{x}^{\prime}, \mathrm{y}^{\prime}\right), \mathrm{B}\left(\mathrm{x}^{\prime}, \mathrm{y}^{\prime}\right), \mathrm{t}\right)\right), 1 / 2\left(\mathrm{~g}\left(\mathrm{M}\left(\mathrm{S}(\mathrm{x}, \mathrm{y}), \mathrm{B}\left(\mathrm{x}^{\prime}, \mathrm{y}^{\prime}\right), \mathrm{t}\right)\right)+\mathrm{g}\left(\mathrm{M}\left(\mathrm{T}\left(\mathrm{x}^{\prime}, \mathrm{y}^{\prime}\right), \mathrm{A}(\mathrm{x}, \mathrm{y}), \mathrm{t}\right)\right)\right)\right\}\right)$ for all $\mathrm{t}>0$ and $\mathrm{x}, \mathrm{y}, \mathrm{x}^{\prime}, \mathrm{y}^{\prime}$ in $\mathrm{X}$, then there exists only one point $\mathrm{b}$ in $\mathrm{X}$ such that $\mathrm{A}(\mathrm{b}, \mathrm{y})=\mathrm{S}(\mathrm{b}, \mathrm{y})=\mathrm{B}(\mathrm{b}, \mathrm{y})=\mathrm{T}(\mathrm{b}, \mathrm{y})$ for all $\mathrm{y}$ in $\mathrm{X}$.

Proof. By (5.1.3),

$\left.\mathrm{g}\left(\mathrm{M}\left(\mathrm{A}(\mathrm{x}, \mathrm{y}), \mathrm{B}\left(\mathrm{x}^{\prime}, \mathrm{y}^{\prime}\right)\right), \mathrm{t}\right)\right) \leq \phi\left(\max \left\{\mathrm{g}\left(\mathrm{M}\left(\mathrm{S}(\mathrm{x}, \mathrm{y}), \mathrm{T}\left(\mathrm{x}^{\prime}, \mathrm{y}^{\prime}\right)\right), \mathrm{t}\right)\right), \mathrm{g}(\mathrm{M}(\mathrm{S}(\mathrm{x}, \mathrm{y}), \mathrm{A}(\mathrm{x}, \mathrm{y})), \mathrm{t})\right)$, $\left.\mathrm{g}\left(\mathrm{M}\left(\mathrm{T}\left(\mathrm{x}^{\prime}, \mathrm{y}^{\prime}\right), \mathrm{B}\left(\mathrm{x}^{\prime}, \mathrm{y}^{\prime}\right)\right), \mathrm{t}\right)\right)$ $\left.\left.\left.\left.1 / 2\left(\mathrm{~g}\left(\mathrm{M}\left(\mathrm{S}(\mathrm{x}, \mathrm{y}), \mathrm{B}\left(\mathrm{x}^{\prime}, \mathrm{y}^{\prime}\right)\right), \mathrm{t}\right)\right)+\mathrm{g}\left(\mathrm{M}\left(\mathrm{T}\left(\mathrm{x}^{\prime}, \mathrm{y}^{\prime}\right), \mathrm{A}(\mathrm{x}, \mathrm{y})\right), \mathrm{t}\right)\right)\right)\right\}\right)$

for all $\mathrm{t}>0$, therefore by Theorem 4.1, for each $\mathrm{y}$ in $\mathrm{X}$, there exists only one $\mathrm{x}(\mathrm{y})$ in $\mathrm{X}$ such that

$\mathrm{A}(\mathrm{x}(\mathrm{y}), \mathrm{y})=\mathrm{S}(\mathrm{x}(\mathrm{y}), \mathrm{y})=\mathrm{B}(\mathrm{x}(\mathrm{y}), \mathrm{y})=\mathrm{T}(\mathrm{x}(\mathrm{y}), \mathrm{y})=\mathrm{x}(\mathrm{y})$ for every $\mathrm{y}, \mathrm{y}^{\prime}$ in $\mathrm{X}$ $\left.\mathrm{g}\left(\mathrm{M}\left(\mathrm{x}(\mathrm{y}), \mathrm{x}\left(\mathrm{y}^{\prime}\right)\right), \mathrm{t}\right)\right)=\mathrm{g}\left(\mathrm{M}\left(\mathrm{A}(\mathrm{x}(\mathrm{y}), \mathrm{y}), \mathrm{A}\left(\mathrm{x}\left(\mathrm{y}^{\prime}\right), \mathrm{y}^{\prime}\right)\right), \mathrm{t}\right)$ $\leq \phi\left(\max \left\{\mathrm{g}\left(\mathrm{M}\left(\mathrm{A}(\mathrm{x}, \mathrm{y}), \mathrm{A}\left(\mathrm{x}^{\prime}, \mathrm{y}^{\prime}\right)\right), \mathrm{t}\right)\right), \mathrm{g}(\mathrm{M}(\mathrm{A}(\mathrm{x}, \mathrm{y}), \mathrm{A}(\mathrm{x}, \mathrm{y})), \mathrm{t})\right)$, $\left.\left.\left.\left.\left.\mathrm{g}\left(\mathrm{M}\left(\mathrm{T}\left(\mathrm{x}^{\prime}, \mathrm{y}^{\prime}\right), \mathrm{A}\left(\mathrm{x}^{\prime}, \mathrm{y}^{\prime}\right)\right), \mathrm{t}\right)\right), 1 / 2\left(\mathrm{~g}\left(\mathrm{M}\left(\mathrm{A}(\mathrm{x}, \mathrm{y}), \mathrm{A}\left(\mathrm{x}^{\prime}, \mathrm{y}^{\prime}\right)\right), \mathrm{t}\right)\right)+\mathrm{g}\left(\mathrm{M}\left(\mathrm{A}\left(\mathrm{x}^{\prime}, \mathrm{y}^{\prime}\right), \mathrm{A}(\mathrm{x}, \mathrm{y})\right), \mathrm{t}\right)\right)\right)\right\}\right)$ $\left.\left.=\mathrm{g}\left(\mathrm{Mx}(\mathrm{y}), \mathrm{x}\left(\mathrm{y}^{\prime}\right)\right), \mathrm{t}\right)\right)$

This implies $\mathrm{x}(\mathrm{y})=\mathrm{x}\left(\mathrm{y}^{\prime}\right)$ and hence $\mathrm{x}(\mathrm{y})$ is some constant $\mathrm{b} \in \mathrm{X}$ so that $\mathrm{A}(\mathrm{b}, \mathrm{y})=\mathrm{b}=\mathrm{T}(\mathrm{b}, \mathrm{y})=\mathrm{S}(\mathrm{b}, \mathrm{y})=\mathrm{B}(\mathrm{b}, \mathrm{y})$ for all $\mathrm{y}$ in $\mathrm{X}$. 


\section{Conclusion}

In this study we prove the common fixed point theorems in Non- Archimedean fuzzy metric space using the concept of semi-compatibility and weak Compatibility of pair of self maps. In fact, the results presented in this paper improve and extend some known results. The previous results such as Chang(1985) are obtained in Probabilistic metric space, while we can get fixed point theorems in more general space.

\section{Acknowledgements}

The author would like to thank the referees for their useful comments and suggestions.

\section{References}

[1] A. George and P. Veeramani, On some results in fuzzy metric space, Fuzzy Sets and Systems, 64(1994), 395-399.

[2] B. Schweizer, H. Sherwood and R. M. Tardi ${ }^{\circledR}$, Contractions on PMspace examples and counterexamples, Stochastica 1 (1988), 5-17.

[3] B. Singh and S. Jain, Semicompatibility and fixed point theorems in fuzzy metric space using implicit relation, 16(2005), 2617-2629.

[4] D. Mihet, A Banach contraction theorem in fuzzy metric spaces, Fuzzy Sets and Systems, 144(2004), 431-439.

[5] D. Mihet, Fuzzy $\psi$-contractive mappings in non-Archimedean fuzzy metric spaces, Fuzzy Sets and Systems 159 (2008) 739 - 744.

[6] I. Kramosil and J. Michalek, Fuzzy metric and statistical metric spaces, Kybernetica, 11(1975), 326-334.

[7] L.A. Zadeh, Fuzzy Sets, Information and Control, 8(1965), 338-353.

[8] L.A. Zadeh, Biological application of the theory of fuzzy sets and systems, in: Proc. Int. Symp. Biocybernetics of the Central Nervous System (Little, Brown \& Co., Boston, 1969) 199-212.

[9] S. S. Chang, On the theory of probabilistic metric spaces with applications, Acta Math. Sinica, New series, 1(4) (1985), 366-377.

[10] S. S. Chang, Fixed point theorems for generalized Meir- Keeler type mappings, J. Sichuan Univ., Natural Science Edition, 2(1983), 17-23.

[11] V. Gregori and A. Sapena, On fixed-point theorem in fuzzy metric spaces, Fuzzy Sets and Sys 125 (2002), 245-252. 